Check for updates

Cite this: RSC Adv., 2017, 7, 50300

\title{
Collagen modified with epoxidized safrole for improving antibacterial activity
}

\author{
Zhou Xu, (D) ab Jinming Chang, ${ }^{a}$ Peikun Zhang, ${ }^{a}$ Xiaoyu Guan, ${ }^{a}$ Yi Chen ${ }^{a}$ \\ and Haojun Fan*a
}

An epoxidized safrole, 5-(oxiran-2-ylmethyl)-benzo[d][1,3]dioxole (OYBD), was synthesized and employed to modify collagen for improving its antibacterial activity. The interaction between collagen and OYBD, and the structure/properties of the modified collagen were investigated in detail. The results indicated that the OYBD-modified collagen showed a higher de-nature temperature (Td, $90.2^{\circ} \mathrm{C}$ ), improved hydrophobic properties (contact angle from $84.2^{\circ}$ to $89.1^{\circ}$ ) and enhanced tensile strength (6.2-11.0\%) without destroying its triple helix structure. From observation of scanning electron microscopy (SEM), a higher density of intertwining morphology and a more stable network structure were observed, which was consistent with improved tensile strength and reduced breaking extension stress. The antibacterial test and LIVE/DEAD Baclight bacterial viability assay illustrated that the modified collagen exhibited excellent antibacterial activity to both Gram-negative and Grampositive bacteria. Furthermore, the OYBD-modified collagen still exhibited cytocompatibility, supporting human fibroblast proliferation, which holds a great potential for developing antibacterial collagen-based biomaterials.

Received 27th July 2017

Accepted 21st October 2017

DOI: $10.1039 / \mathrm{c} 7 \mathrm{ra0} 8319 \mathrm{j}$

rsc.li/rsc-advances identified as being of importance for its functional and bioactive properties.

Previous studies demonstrated that collagen cross-linked with epoxy compounds displayed comparable mechanical properties to those cross-linked by glutaraldehyde. ${ }^{5}$ Additionally, cross-linking with epoxy compound showed no signs of cytotoxic degradation products. ${ }^{\mathbf{9} 10}$ To improve the antibacterial activity of collagen, we synthesized a new modifier in this study, named 5-(oxiran-2-ylmethyl)-benzo[ $d][1,3]$ dioxole (OYBD), derived from natural safrole (4-allyl-1,2-(methylenedioxy)benzene). This modifier contained an epoxy group and a piperonyl group. The former could react with amine groups of collagen, and the latter was known as antibacterial. After modification, the collagen would be expected to yield desirable antibacterial activity as well as enhanced thermal and mechanical properties.

\section{Experimental}

\subsection{Materials}

Pigskins were obtained from a local tannery. Safrole $(\geq 97 \%)$ was purchased from Sigma-Aldrich (St. Louis, MO) and used as received. Pepsin (3000-3500 $\mathrm{U} \mathrm{g}^{-1}$ ), MTT (dimethyl thiazolyl tetrazolium bromide), and 3-chloroperbenzoic acid (mCPBA) were purchased from Sino Standards Biotechnology Co. Ltd. (Chengdu, China). Dichloromethane, $\mathrm{NaOH}, \mathrm{MgSO}_{4}, \mathrm{NaCl}$, and HAc were obtained from Kelong Chemical Engineering Co. Ltd. (Chengdu, China). All chemicals were of analytical grade or biological reagent grade.
${ }^{a}$ Key Laboratory of Leather Chemistry and Engineering of Ministry of Education, Sichuan University, Chengdu 610065, China. E-mail: fanhaojun@scu.edu.cn ${ }^{b}$ School of Life Science and Food Engineering, Yibin University, Yibin 644007, China 


\subsection{Synthesis of 5-(oxiran-2-ylmethyl)-benzo $[d][1,3]$ dioxole (OYBD)}

The synthesis of OYBD was conducted according to the following procedure. $m$-chloroperbenzoic acid $(m \mathrm{CPBA})(31.8 \mathrm{~g}$, $0.15 \mathrm{~mol}$ ) was first dissolved in $200 \mathrm{~mL}$ of dichloromethane (DCM), followed by addition of safrole $(16.2 \mathrm{~g}, 0.1 \mathrm{~mol})$ at room temperature. After stirred for $24 \mathrm{~h}$, the mixture was filtered, and the precipitate was discarded. The supernatant was washed with $5 \% \mathrm{NaOH}$ solution three times and subsequently with deionized water three times. The organic layer obtained was dried over anhydrous magnesium sulfate $\left(\mathrm{MgSO}_{4}\right)$. The solvent was removed by distillation $\left(110{ }^{\circ} \mathrm{C}, 530 \mathrm{~Pa}\right)$, and OYBD was finally obtained with a yield of $82 \%$.

\subsection{Preparation of native collagen}

The pig skin was first pulverized with a mill (Fristsch puluterisette, ldar-Oberstein, Germany) and then suspended in a mixture of $0.5 \mathrm{M} \mathrm{HAc}(3 \%, \mathrm{w} / \mathrm{v})$ and pepsin $(2 \%, \mathrm{w} / \mathrm{w})$ at $4{ }^{\circ} \mathrm{C}$ for $48 \mathrm{~h}$ under magnetic stirring. After that, the supernatant was collected through centrifugation at $10000 \mathrm{rpm}$ for $10 \mathrm{~min}\left(4^{\circ} \mathrm{C}\right)$ and then salted using excessive $\mathrm{NaCl}$. The precipitate was subsequently dialyzed against $0.1 \mathrm{M} \mathrm{HAc}$ at $4{ }^{\circ} \mathrm{C}$ for 3 days to obtain native collagen. The collagen was lyophilized using a lyophilized at $-50{ }^{\circ} \mathrm{C}$ for $48 \mathrm{~h}$ for further use.

\subsection{Modification of collagen by OYBD}

About $0.5 \mathrm{~g}$ native collagen was first dissolved in $0.5 \mathrm{M} \mathrm{HAc}$. The mixture was cast to form an aqueous membrane, which was then dried at $37{ }^{\circ} \mathrm{C}$ until a constant weight. The resultant collagen membrane was immersed in $100 \mathrm{~mL}$ of an ethanesulfonic acid (MES, TRC, Canada) buffer solution $(\mathrm{pH}=8.0)$ containing $5 \mathrm{wt} \%$ OYBD. The epoxidation reaction was conducted at room temperature for $36 \mathrm{~h}$. After that, the collagen membrane was rinsed in phosphate buffer saline for $24 \mathrm{~h}$ and washed with deionized-water five times. The resultant material was denoted as collagen-OYBD.

\section{$2.5 \quad{ }^{1} \mathrm{H}$ NMR detection}

The ${ }^{1} \mathrm{H}$ NMR spectrum of OYBD was recorded by a NMR spectrometer (AV11-600MH, Bruker, Swiss) with a test frequency of $600 \mathrm{MHz}$. The trifluoroacetic acid-D (99.5 atom\% D) was used as deuterium reagent, and tetramethylsilane (TMS) as internal standard.

\subsection{FTIR spectra measurement}

Fourier transform infrared (FTIR) spectra were collected using a Nicolet iS10 FTIR spectrometer (Thermo Fisher Scientific, United States) over a wavenumber range from 500 to $4000 \mathrm{~cm}^{-1}$ after 64 scans at a resolution of $4 \mathrm{~cm}^{-1}$.

\subsection{DSC measurement}

Briefly, $3 \mathrm{mg}$ collagen specimens were loaded in an aluminum pan. The sample pan and the empty reference aluminum pan were both placed in a Netzsch DSC 200 PC operating in $\mathrm{N}_{2}$ flow.
Differential scanning calorimetry (DSC) analysis was conducted in the temperature range from 20 to $180{ }^{\circ} \mathrm{C}$ with a heating rate of $4{ }^{\circ} \mathrm{C} \min ^{-1}$.

\subsection{SEM observation}

The morphology of the lyophilized collagen samples $(1 \mathrm{~cm} \times 1$ $\mathrm{cm}$ ) was observed with a scanning electron microscopy (SEM, Nova Nano SEM450, FEI, USA). The collagen specimen was sputter-coated with aurum and imaged at an accelerating voltage of $5 \mathrm{kV}$.

\subsection{Water contact angle (WCA) test}

The hydrophilicity of the OYBD-modified collagen was measured using a goniometer (Dataphysics, OCAH200, Germany) at room temperature. Briefly, $5 \mu \mathrm{L}$ per drop of distilled water was drop-wise deposited on the surface of the collagen, and the WCA was calculated using a shape DSA100 analysis system. Each sample was tested for five different surface areas to obtain an average value.

\subsection{Mechanical measurement}

The collagen membrane was cut into a dumbbell shape with length and width of $50.0 \mathrm{~mm} \times 10 \mathrm{~mm}$ using a dumbbellshaped knife. The thickness of the samples was measured at three different areas using a micrometer, and an average value was calculated. Using a universal tensile testing machine (GTAI-7000S, Hi tech, Taiwan), a crosshead speed of $1 \mathrm{~mm} \mathrm{~min}^{-1}$ was applied until the collagen membrane ruptured. The tensile strength, elongation at maximum force, and elongation at break of the collagen sample were calculated, and each sample was measured three times.

\subsection{Antibacterial measurement}

Escherichia coli (E. coli, ATCC 25922) was selected as the indicator in evaluating the antibacterial activity of the OYBDmodified collagen. A small quantity of recovered cultures of E. coli was diluted with saline to a concentration of $1 \times 10^{8}$ colony-forming unit (cfu) per mL. Subsequently, $2 \mathrm{~mL}$ of $E$. coli suspensions were transferred into a tube containing $18 \mathrm{~mL}$ of sterile water, followed by addition of $0.15 \mathrm{~g}$ collagen membrane that had been subject to irradiation sterilization. Furthermore, the suspension was shook for $1 \mathrm{~h}$. Afterwards, $1 \mathrm{~mL}$ of the resulting mixture was incorporated into $9 \mathrm{~mL}$ of sterile water to produce a series of dilution. For each diluted tube, $0.1 \mathrm{~mL}$ of the bacterial suspension was evenly distributed onto a sterilized nutrient agar Petri dish and incubated for $48 \mathrm{~h}$ in an incubator at $37^{\circ} \mathrm{C}$. The number of viable micro-organisms colony and the antibacterial ratio were measured.

$$
\text { Antibacterial rate }(\%)=\frac{w_{1}-w_{2}}{w_{1}} \times 100 \%
$$

where, $w_{1}$ is the cfu of collagen and $w_{2}$ is the cfu of the OYBDmodified collagen. 


\subsection{LIVE/DEAD Baclight bacterial viability test}

The LIVE/DEAD Baclight bacterial viability kit was used to visualize the bacterial cells on the modified collagen. The fluorescent dyes agents of green stain SYTO 9 (label both live and dead cells) and red stain propidium iodide (only penetrate cells with compromised or damaged membranes) could bind to nucleic acids to assay cell viability. A suspension of Staphylococcus aureus ( $S$. aureus, ATCC 25923$)$ in PBS $\left(10^{5} \mathrm{CFU} \mathrm{mL}^{-1}, 50\right.$ $\mu \mathrm{L})$ was added onto the collagen samples, ${ }^{11}$ which was then incubated for $24 \mathrm{~h}$ at $35{ }^{\circ} \mathrm{C}$. Subsequently, the collagen sample was washed with PBS buffer and soaked in SYTO $9(4 \mu \mathrm{L})$ and propidium iodide $(6 \mu \mathrm{L})$ dye solution in dark condition for $15 \mathrm{~min}$. Finally, the stained bacterial cells were observed using an IX-71 inversed fluorescent microscope (Olympus America Inc., Melville, NY).

\subsection{Cytocompatibility analysis}

The cell proliferation assay was carried out to evaluate the cytocompatibility of the modified collagen according to ISO 10993-5. Normal human dermal fibroblasts (NHDFs) were seeded in a culture dish and cultured to near confluence at $37{ }^{\circ} \mathrm{C}$ under $5 \% \mathrm{CO}_{2}$. Collagen solution was diluted to a concentration of $20 \mu \mathrm{g} \mathrm{mL}^{-1}$ with $0.05 \mathrm{M}$ acetic acid solution, and then added into the wells of a 24 -well polystyrene plate. After incubation for $12 \mathrm{~h}$, the solution was neutralized with 0.05 M sodium hydroxide solution, and the wells were rinsed with PBS three times. Subsequently, NHDFs $\left(1 \times 10^{5}\right.$ cells $)$ in 2 mL Dulbecco's Modified Eagle's Medium (DMEM) with 10\% fetal bovine serum were seeded. After incubation at $37{ }^{\circ} \mathrm{C}$ with $5 \% \mathrm{CO}_{2}$ for 1,3 , and $7 \mathrm{~d}$ respectively, cell proliferation was measured using the methyl thiazolyl tetrazolium (MTT) assay. In addition, the morphologies of cells were observed by employing an IX83 2-deck inverted microscope (Olympus).

\subsection{Statistical analysis}

The reported data are the means \pm standard deviation of triplicate samples for each measurement. Statistical analysis was performed with Student's $t$-test. $P<0.05$ was accepted as statistically significant.

\section{Results and discussion}

\subsection{Cross-linking between collagen and OYBD}

The ${ }^{1} \mathrm{H}$ NMR measurement was performed to confirm the structure of 5-(oxiran-2-ylmethyl)benzo[d][1,3]dioxole (OYBD) and the results were shown in Fig. 1 . Safrole was oxidized by the weak oxidant ( $m$ CPBA), forming a monofunctional epoxy group, which could react with those amino groups originated from collagen. The ${ }^{1} \mathrm{H}$ NMR detection results of OYBD are as follows: ${ }^{1} \mathrm{H}$ NMR (600 MHz, DMSO- $d_{6}$ ) $\delta: 6.91-6.66$ (m, 3H, ArCH), 5.98 (s, $\left.2 \mathrm{H},-\mathrm{OCH}_{2} \mathrm{O}^{-}\right), 3.06$ (dp, $J=5.9,3.0 \mathrm{~Hz}, 1 \mathrm{H},-\mathrm{OCH}\left(\mathrm{CH}_{2}\right) \mathrm{CH}_{2}-$ ), 2.79-2.61 (m, $3 \mathrm{H},-\mathrm{ArCH}_{2}-\mathrm{CH}$ and $\left.-\mathrm{OCH}_{2} \mathrm{CH}\right), 2.53(\mathrm{dd}, J=5.1$, $\left.2.6 \mathrm{~Hz}, 1 \mathrm{H},-\mathrm{OCH}_{2} \mathrm{CH}\right)$. Such result is consistent with that as previously reported. ${ }^{12,13}$

Possible interactions between collagen and OYBD are illustrated in Fig. 2. According to previous studies, collagen can be
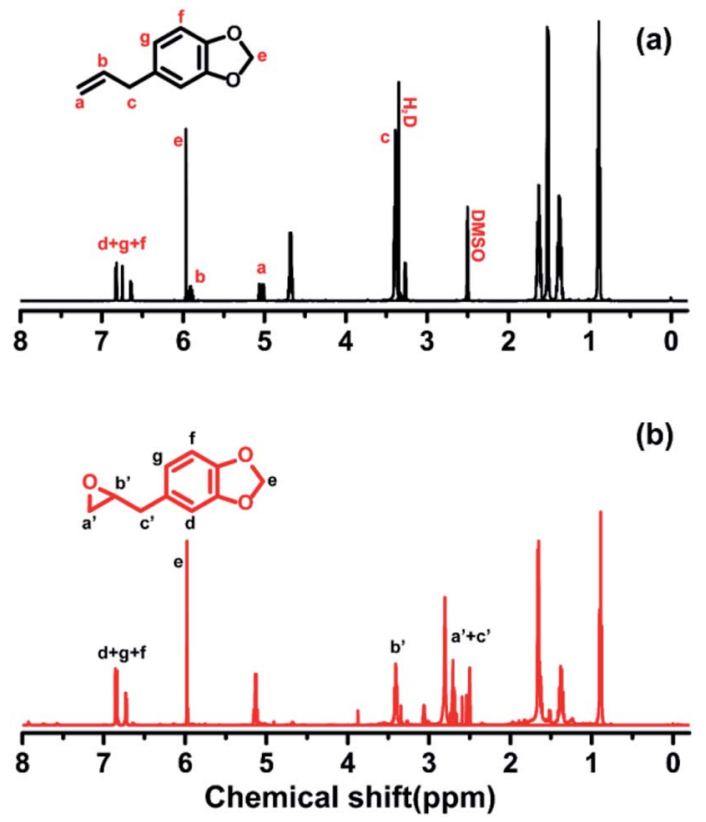

Fig. $1{ }^{1} \mathrm{H}$ NMR spectra of (a) safrole and (b) 5-(oxiran-2-ylmethyl) benzo[d][1,3]dioxole.

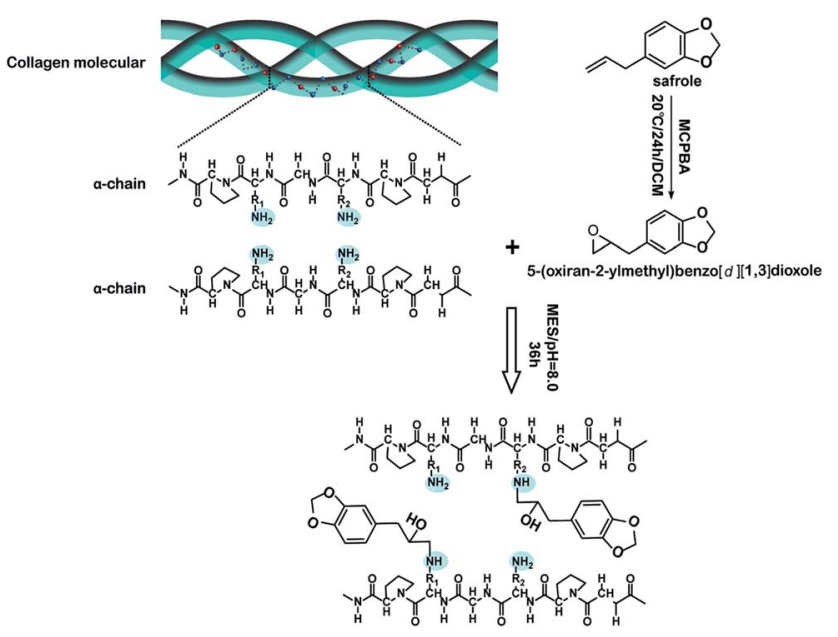

Fig. 2 Schematic illustration of possible interactions between collagen and OYBD.

modified by monofunctional and multifunctional poly epoxy compounds under alkaline condition. ${ }^{14}$ The synthesized OYBD contained an epoxidized structure that could react with the amine groups of lysine residues in collagen under alkaline condition ( $\mathrm{pH}$ 8.0). Thus, the antibacterial piperonyl group can be covalently introduced into the collagen macromolecules.

\subsection{The triple-helical structure retention of collagen}

An ideal modification strategy should not destroy the special triple-helical structure of tropocollagen molecules that is the basis of many important and specific biological functions of collagen. ${ }^{15,16}$ In general, the FTIR absorption band at $1235 \mathrm{~cm}^{-1}$ 
corresponds to the $\mathrm{C}-\mathrm{N}$ bond in-plane vibration (amide III) and the $\mathrm{N}-\mathrm{H}$ stretch (amide I), which is associated with the triplehelical structure of tropocollagen molecules. The absorption band at $1450 \mathrm{~cm}^{-1}$ can be ascribed to the pyrrolidine ring vibration of proline and hydroxyproline, which is not affected by changes in collagen secondary structure. Thus, it is widely accepted that the intensity ratio of FTIR absorption at 1235 and $1450 \mathrm{~cm}^{-1}\left(I_{\left.1235 / 1450 \mathrm{~cm}^{-1}\right)}\right.$ can be used as a sensitive indicator of the integrity of triple-helical structure in collagen. ${ }^{17}$ Typically, an $I_{1235 / 1450 \mathrm{~cm}^{-1}}$ close to unity indicates intact triple-helical structure, whereas an $I_{1235 / 1450 \mathrm{~cm}^{-1}}$ significantly lower than unity suggests the triple helicity has been completely destroyed. ${ }^{18}$

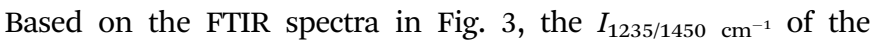
OYBD-modified collagen was 0.91 and is comparable to the value of native collagen (1.0). This result revealed that the triple helix conformation was not destroyed in the modification process by OYBD.

In addition, quantitative analysis of molar ellipticity changes in $\mathrm{CD}$ spectra before and after OYBD treatment could also provide valuable information regarding the integrity of triplehelical structure. CD spectroscopy is the most widespread technique used for estimating the secondary structures of proteins and polypeptides in solution. This technique can be used to distinguish between unordered (random coil) and ordered (triple helix) structures of collagen. CD detects wavelength-dependent differences in the absorption of right and left circularly polarized light by optically active molecules such as collagen. The CD spectrum of unordered collagen is usually characterized by a single band below $200 \mathrm{~nm}$, whereas triple-helical structure usually presents one negative band at $223 \mathrm{~nm}$, along with one positive band at $197 \mathrm{~nm} .{ }^{19,20}$ It has been well established that the absolute ratio value of molar ellipticity at $220 \mathrm{~nm}$ over molar ellipticity around 195-197 nm (Rpn) can be used to characterize the integrity of triple-helical conformation of collagen in solution. As shown in Fig. 4, the OYBD modified collagen exhibited a positive peak at $223 \mathrm{~nm}$ and a negative peak at $197 \mathrm{~nm}$, respectively. Additionally, the Rpn value of the OYBD-modified collagen (0.13) was comparable to

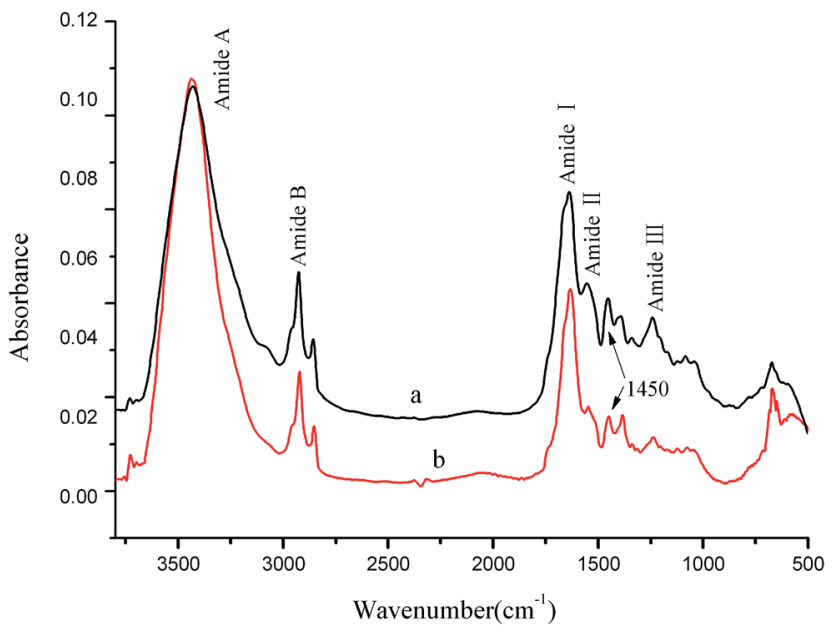

Fig. 3 FTIR spectra of (a) native collagen and (b) OYBD-modified collagen.

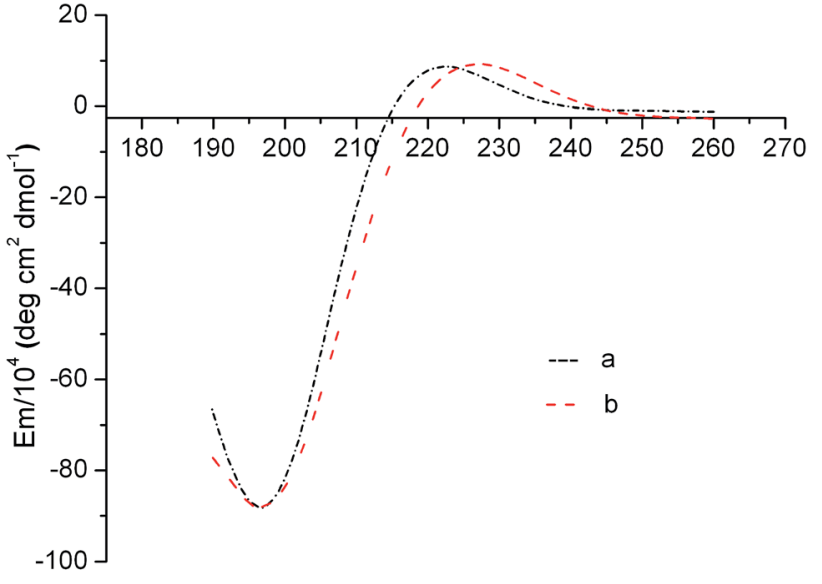

Wavelength $(\mathrm{nm})$

Fig. 4 CD spectra of (a) native collagen and (b) OYBD-modified collagen.

that of the native collagen (0.14), suggesting that modification by OYBD did not destroy the triple helix structure of collagen. This conclusion is well consistent with FTIR analysis.

\subsection{De-nature temperature of collagen}

Differential scanning calorimetry (DSC) is used to analyze the endothermic shrinkage transition of collagen. As shown in Fig. 5, the OYBD-modified collagen showed higher de-nature temperature $\left(90.2{ }^{\circ} \mathrm{C}\right)$ than native collagen $\left(69.9^{\circ} \mathrm{C}\right)$. This phenomenon occurred because OYBD reacted with $-\mathrm{NH}_{2}$ from lysine or hydroxylysine side groups of collagen and, forming a more stable structure, which enhanced the thermal stability of collagen. ${ }^{21}$

\subsection{Microstructure appearance of collagen}

The morphology and porosity of porous materials are very important for the scaffolds. The morphology of collagen

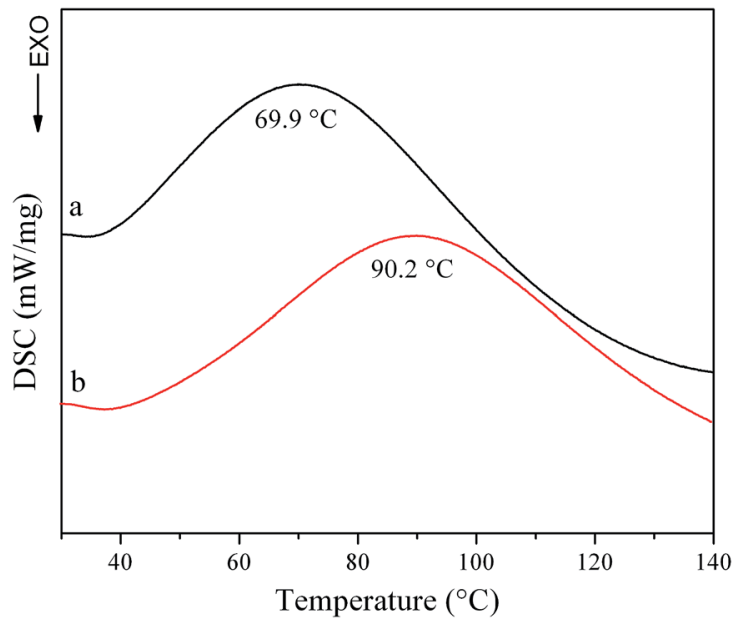

Fig. 5 Differential scanning calorimetry curves of (a) native collagen and (b) OYBD-modified collagen. 
membrane was observed by SEM. As shown in Fig. 6, the fibers of the OYBD-modified collagen exhibited an intertwining morphology and more dense, intensive fiber structure under $\times 100, \times 1200$, and $\times 5000$ magnification compared with native collagen, indicating efficient crosslinking between collagen and OYBD.

\subsection{Hydrophobic-hydrophilic properties of collagen}

The hydrophobic/hydrophilic properties of collagen can be evaluated by the value of water contact angle (WCA). In general, a low value of WCA means higher hydrophilicity, whilst a high value of WCA indicates better hydrophobicity. ${ }^{22}$ As shown in Fig. 7, the value of WCA is $89.1 \pm 1.4^{\circ}$ for OYBD-modified collagen and $84.2 \pm 0.8^{\circ}$ for native collagen, suggesting that OYBD modification increased the hydrophobicity of collagen. The underlying mechanism is that a certain amount of the hydrophobic phenoxy groups is introduced into the collagen side chains in the modification process, which leads stronger water repellence of the surface, and thus hydrophobicity. An increased hydrophobic property of the OYBD-modified collagen is believed to be favorable in preventing water and bacteria intrusion for collagen-based biomaterials.
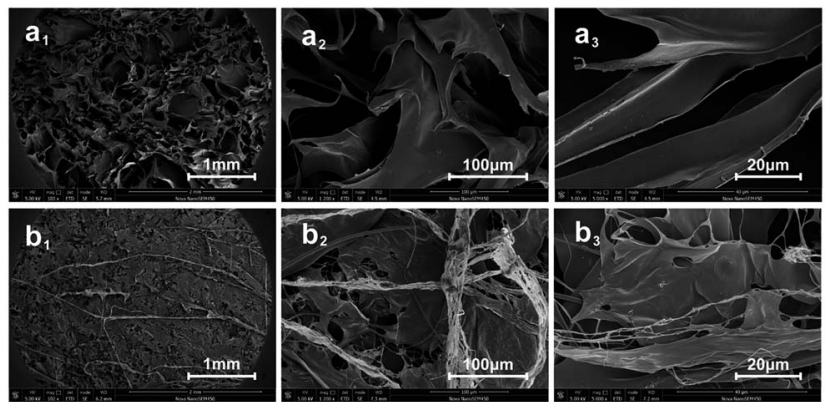

Fig. 6 SEM images of $\left(a_{1}\right),\left(a_{2}\right)$, and $\left(a_{3}\right)$ native collagen and $\left(b_{1}\right),\left(b_{2}\right)$, and $\left(b_{3}\right)$ OYBD-modified collagen under different magnifications.

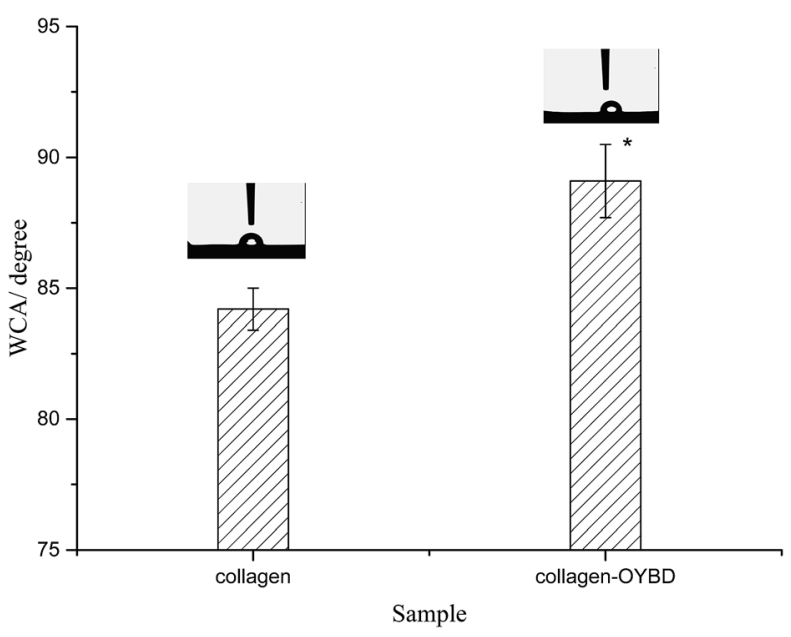

Fig. 7 Water contact angle of native collagen and OYBD-modified collagen. Statistical significance: $* p<0.05$. The reported data are mean \pm S.D. $(N=3)$.

\subsection{Mechanical properties of collagen}

Cross-linking can efficiently increase the mechanical properties of the collagen-based materials, especially, the tensile strength and strain modulus. ${ }^{23}$ As illustrated in Fig. 8, collagen crosslinked with OYBD at $\mathrm{pH} 8.0$ had exhibited increased tensile strength (42.9 $\mathrm{MPa})$ and elongation at break compared with native collagen. The enhanced tensile strength of the modified collagen may be related to the nature of inter-microfibrillar cross-links. ${ }^{14}$ As shown in the SEM images in Fig. 6, the modified collagen displays a more dense microfibrillar structure which suggests that the OYBD-induced cross-links hinder the slippage of collagen fibrils and consequently creates internal stresses in the fibers. Thus, the modification with OYBD increases the mechanical properties of the collagen.

\subsection{Antibacterial activity of collagen}

As shown in Fig. 9, the colony-forming unit (cfu) of all dilution from the modified collagen is less than those from the native collagen. The antibacterial ratio of the OYBD-modified collagen is $51.6 \%$ when the suspension was diluted to $1: 100$, while the antibacterial ratio reached up to $100 \%$ at the dilution of $1: 1000$. The antibacterial activity against $E$. coli is the result of the unique piperonyl structures from OYBD. This structure is suggested to be very important in many compounds with physiological activity. ${ }^{24}$ For E. coli, a type of typical Gramnegative bacteria, the inhibitory activity of the OYBD-modified collagen is attributed to the destruction of the peptidoglycan layer and lipo-polysaccharide. More interestingly, the antibacterial activity of the OYBD-modified collagen for Gram-negative bacteria has a potential application in collagen based biomaterials.

The LIVE/DEAD Baclight bacterial viability test is another analytical method to detect the antibacterial activity of both Gram-negative and Gram-positive bacterial. The green stain SYTO 9 is particularly useful as a nuclear counter stain for

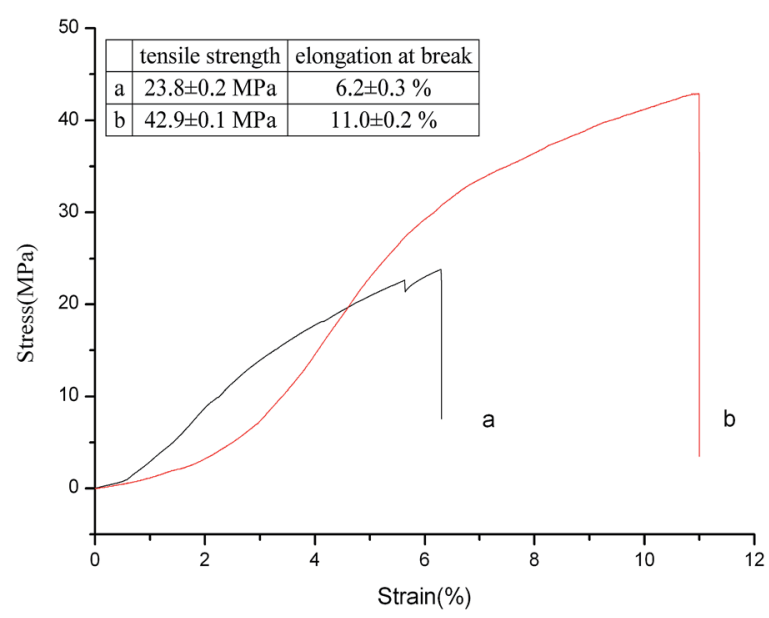

Fig. 8 Typical uniaxial stress-strain curves of (a) native collagen and (b) OYBD-modified collagen $\left(20^{\circ} \mathrm{C}\right.$, distance between clamps: $1 \mathrm{~cm}$, pull speed: $\left.1 \mathrm{~mm} \mathrm{~min}^{-1}, N=3\right)$. The reported data are mean \pm S.D. $(N=3)$. 

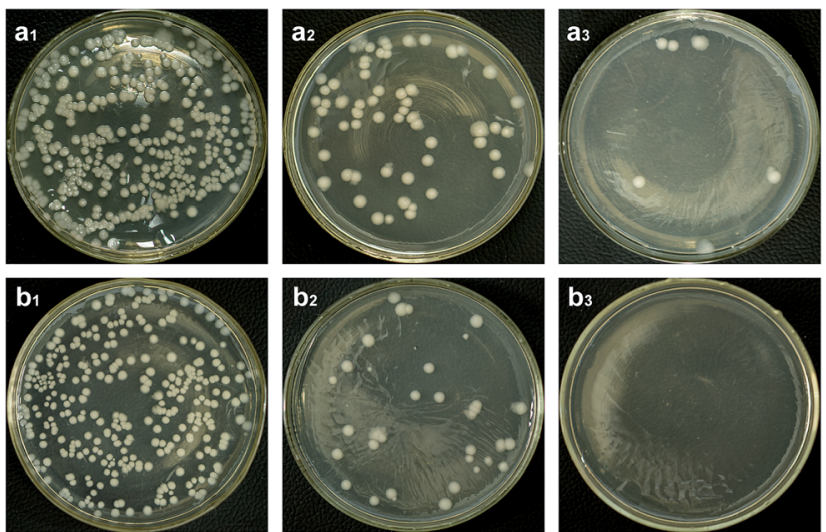

Fig. 9 The cfu of (a) native collagen and (b) OYBD-modified collagen $a_{1}\left(b_{1}\right), a_{2}\left(b_{2}\right)$ and $a_{3}\left(b_{3}\right)$ represent the dilution of $10^{-1}, 10^{-2}$ and $10^{-3}$

bacterial assays since it can stain both live and dead Grampositive bacteria. ${ }^{25}$ The red stain propidium iodide (PI) can only penetrate cells with compromised or damaged membranes. As shown in Fig. 10, the predominance of green fluorescence (live bacteria) on native collagen after dyed by SYTO 9 and PI indicated most of the seeded S. aureus bacteria remained viable, which suggested the collagen and/or polypeptide, amino acid are beneficial for bacterial growth. Nevertheless, little green fluorescence could be found on collagenOYBD after stained by SYTO 9 and PI, which suggested that the collagen-OYBD remained antibacterial activity for Grampositive bacteria.

\subsection{Cytocompatibility assay}

With intact triple-helical confirmation, the biological function of collagen modified with OYBD remained uncompromised. The morphologies of human fibroblasts cultured in collagencoated PS wells for three days are illustrated in Fig. 11(1). Clearly, the cells seeded on OYBD-modified collagen showed
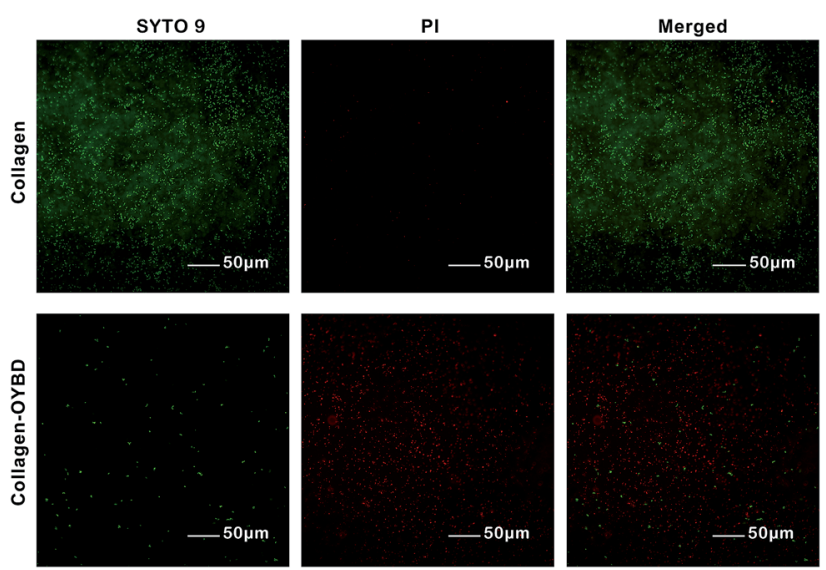

Fig. 10 Fluorescent images of $S$. aureus seeded on native collagen and OYBD-modified collagen (green, live bacteria; red, dead bacteria). The merged images represent the superposition of live and dead bacteria.
(1)
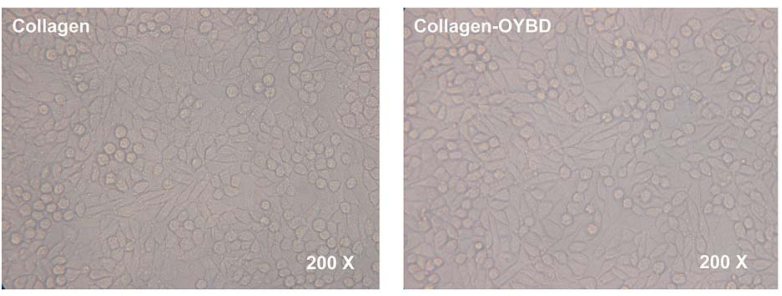

(2)

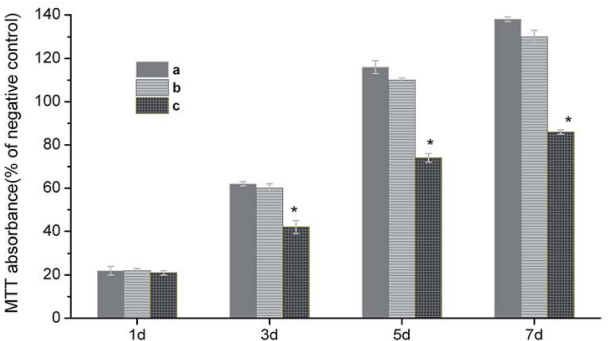

Fig. 11 (1) The morphology of fibroblasts on the culture plates at day 3; (2) cytotoxicity tests from MTT assays of cell viability over a period of 7 days ((a) collagen, (b) OYBD-modified collagen, (c) gelatin). Statistical significance: $* p<0.05$. The reported data are mean \pm S.D. $(N=3)$.

similar morphologies to those on native collagen, exhibiting typical fibroblast morphologies, a spindle shape. This observation demonstrated that the modified collagen supported attachment and differentiation of human fibroblasts as efficiently as native collagen. In addition, the fibroblast proliferation rate was also quantitatively evaluated by using the MTT assay. As illustrated in Fig. 11(2), human fibroblast not only remained viable, but also proliferated on the OYBD-modified collagen, significantly faster than those on gelatin-coated wells for the same seeding time. This was because gelatin has no triple-helical confirmation, ${ }^{26}$ and thus did not act as an efficient support for cell attachment and proliferation.

\section{Conclusions}

Modification of collagen with epoxidized safrole, 5-(oxiran-2ylmethyl)-benzo[ $d][1,3]$ dioxole (OYBD) under alkaline condition resulted in a modified collagen with increased thermal stability, enhanced mechanical properties, improved hydrophobic properties together with more density intermicro-fibrillar structure. Especially, the introduction of OYBD did not destroy the triple helix conformation of native collagen according to FTIR and CD analysis. Additionally, antibacterial tests revealed that the modified collagen inhibited proliferation of both Gram-negative bacterial E. coli and Gram-positive bacteria $S$. aureus efficiently. Simultaneously, the collagenOYBD exhibited excellent cytocompatibility. According to these results, OYBD holds a great potential as a novel modifier for producing antibacterial collagen-based biomaterials.

\section{Conflicts of interest}

There are no conflicts to declare. 


\section{Acknowledgements}

The authors wish to acknowledge the financial support from National Natural Science Foundation of China (No. 51273128, 21206096) and National Key R\&D Program of China (No. 2017YFB 0308600).

\section{References}

1 R. Zeeman, P. J. Dijkstra and P. B. Van Wachem, J. Biomed. Mater. Res., 1999, 46, 424-433.

2 D. M. Simmons and J. N. Kearney, Biotechnol. Appl. Biochem., 1993, 17, 23-29.

3 R. Tu, S. H. Shen and D. Lin, J. Biomed. Mater. Res., 1994, 28, 677-684.

4 J. M. Lee, C. A. Pereira and L. W. K. Kan, J. Biomed. Mater. Res., 1994, 28, 981-992.

5 T. Xi and F. Liu, J. Biomater. Appl., 1992, 7, 61-75.

6 R. Sripriya, R. Kumar, S. Balaji, M. S. Kumar and P. K. Sehgal, React. Funct. Polym., 2011, 71, 62-69.

7 L. H. H. Olde Damink, P. J. Dijkstra, M. J. A. van Luyn, P. B. van Wachem, P. Nieuwenhuis and J. Feijen, J. Mater. Sci.: Mater. Med., 1995, 6, 429-434.

8 R. Tu, R. C. Quijano, C. L. Lu, et al., Int. J. Artif. Organs, 1993, 16, 537-544.

9 J. M. Lee, C. A. Pereira and L. W. K. Kan, J. Biomed. Mater. Res., 1994, 28, 981-992.

10 J. M. Lohre, L. Baclig, J. Sagartz, S. Guida, K. Thyagarajan and R. Tu, Artif. Organs, 1992, 16, 630-633.

11 R. Garcíapumarino, G. Pascual, M. Rodríguez, et al., J. Biomed. Mater. Res., Part B, 2013, 102, 366-375.
12 L.-Y. Wang, X.-H. Wang and J.-L. Tan, Bull. Korean Chem. Soc., 2012, 33, 3571-3575.

13 B. X. Zhao, D. W. Wang, H. Zuo, G. T. Qian and J. Y. Miao, Chin. J. Org. Chem., 2003, 23, 1026-1028.

14 R. Zeeman, P. J. Dijkstra and P. B. V. Wachem, Biomaterials, 1999, 20, 921-931.

15 H. Zhou, R. P. Xun, Z. H. Zhou, Q. Q. Liu, P. Wu and K. J. Wu, Fibers Polym., 2014, 15, 519-524.

16 M. E. Andrews, J. Murali, C. Muralidharan, W. Madhulata and R. Jayakumar, Colloid Polym. Sci., 2003, 281, 766-770.

17 L. He, C. Mu, J. Shi, Q. Zhang, B. Shi and W. Lin, Int. J. Biol. Macromol., 2011, 48, 354-359.

18 B. Madhan, V. Subramanian and J. R. Rao, Int. J. Biol. Macromol., 2005, 37, 47-53.

19 D. I. Zeugolis, S. T. Khew, E. S. Yew, et al., Biomaterials, 2008, 29, 2293.

20 J. Chang, Y. Chen, S. Zhao, et al., Polym. Chem., 2015, 6, 8150-8160.

21 B. Balakrishnan, M. Mohanty, P. R. Umashankar and A. Jayakrishnan, Biomaterials, 2005, 26, 6335-6342.

22 P. Fratzl, Collagen: Structure and Mechanics, Springer, New York, NY, USA, 2008.

23 P. F. Gratzer and J. M. Lee, J. Biomed. Mater. Res., 1997, 37, 497-507.

24 D. Ge, Q. Jing, W. Zhao, H. Yue, et al., PLoS One, 2014, 9, e99378.

25 C. Yang, X. Ding, R. J. Ono, et al., Adv. Mater., 2014, 26, 73467351.

26 G. Y. Li, S. Fukunaga, K. Takenouchi and F. Nakamura, Int. J. Cosmet. Sci., 2005, 27, 101. 